\title{
GLOBAL DYNAMICS IN THE POINCARÉ BALL OF THE CHEN SYSTEM HAVING INVARIANT ALGEBRAIC SURFACES
}

\author{
JAUME LLIBRE \\ Departament de Matemàtiques, Universitat Autònoma de Barcelona, \\ 08193 Bellaterra, Barcelona, Catalonia, Spain. E-mail: jllibre@mat.uab.cat \\ MARCELO MESSIAS* \\ Departamento de Matemática, Estatística e Computação, \\ Faculdade de Ciências e Tecnologia, UNESP - Univ Estadual Paulista, Cx. Postal 266, \\ 19060-900, Presidente Prudente, São Paulo,Brazil. E-mail: marcelo@fct.unesp.br \\ PAULO RICARDO DA SILVA \\ Departamento de Matemática, Instituto de Biociências, Letras e Ciências Exatas, \\ UNESP - Univ Estadual Paulista, Rua C. Colombo, 2265, \\ 15054-000, S. J. Rio Preto,São Paulo,Brazil.E-mail: prs@ibilce.unesp.br
}

In this paper we perform a global analysis of the dynamics of the Chen system

$$
\dot{x}=a(y-x), \quad \dot{y}=(c-a) x-x z+c y, \quad \dot{z}=x y-b z,
$$

where $(x, y, z) \in \mathbb{R}^{3}$ and $(a, b, c) \in \mathbb{R}^{3}$. We give the complete description of its dynamics on the sphere at infinity. For six sets of the parameter values the system has invariant algebraic surfaces. In these cases we provide the global phase portrait of the Chen system and give a complete description of the $\alpha$ - and $\omega$-limit sets of its orbits in the Poincaré ball, including its boundary $\mathbb{S}^{2}$, i.e. in the compactification of $\mathbb{R}^{3}$ with the sphere $\mathbb{S}^{2}$ of the infinity. Moreover, combining the analytical results obtained with an accurate numerical analysis, we prove the existence of a family with infinitely many heteroclinic orbits contained on invariant cylinders when the Chen system has a line of singularities and a first integral, which indicates the complicated dynamical behavior of the Chen system solutions even in the absence of chaotic dynamics.

Keywords: Chen system, integrability, Poincaré compactification, dynamics at infinity, heteroclinic orbits, singularly degenerate heteroclinic cycles, invariant manifolds.

\section{Introduction and statement of the main results}

The Chen system is the three-parameter family of quadratic polynomial differential equations given by

$$
\dot{x}=P=a(y-x), \quad \dot{y}=Q=(c-a) x-x z+c y, \quad \dot{z}=R=x y-b z,
$$

with the state variables $(x, y, z) \in \mathbb{R}^{3}$ and the parameters $(a, b, c) \in \mathbb{R}^{3}$. As usual the dots denote differentiation with respect to the time $t$.

${ }^{*}$ Author for correspondence 
System (1) was firstly studied in [Chen and Ueta, 1999]. It exhibits chaotic phenomena for suitable choices of the values of the real parameters $a, b$ and $c$. In this way, some important properties of system (1) are similar to the properties of the well-known Lorenz system [Lorenz, 1963]

$$
\dot{x}=r y-x-y z, \quad \dot{y}=\sigma(x-y), \quad \dot{z}=-b z+x y .
$$

For instance, one can easily check that: Systems (1) and (2) are invariant under the change of variables $(x, y, z) \longmapsto(-x,-y, z)$, consequently if $(x(t), y(t), z(t))$ is a solution of system (1) or (2), then $(-x(t),-y(t), z(t))$, its symmetric with respect to the $z$-axis, is also a solution. In [Lu et al., 2002] the authors introduce a unified chaotic system containing the Lorenz and the Chen systems as dual systems at the two extremes of its parameter spectrum. This unified system represents the transition from the Lorenz to the Chen system and is chaotic over the entire range of the parameter.

Let $\mathbb{R}[x, y, z]$ be the ring of the real polynomials in the variables $x, y$ and $z$. We say that $F=F(x, y, z) \in$ $\mathbb{R}[x, y, z]$ is a Darboux polynomial of system (1) if it satisfies $(\nabla F) \cdot(P, Q, R)=k F$, where $k=k(x, y, z)$ is a real polynomial of degree at most 1 , called the cofactor of $F(x, y, z)$, and $\nabla F$ denotes the gradient of $F$. If the cofactor is zero, then $F(x, y, z)$ is a polynomial first integral of system (1). If $F(x, y, z)$ is a Darboux polynomial, then the surface $F(x, y, z)=0$ is an invariant algebraic surface; i.e. if an orbit of system (1) has a point on this surface, then the whole orbit is contained in it. The following proposition gives a summary on the invariant algebraic surfaces of system (1), it is due to Lu and Zhang [Lu and Zhang, 2007].

Proposition 1.1. If $a=0$ then the phase portrait of system (1), restricted to each plane $x=$ constant is determined by a linear differential system. If $a \neq 0$ then system (1) has the invariant algebraic surfaces $F_{i}=0, i=1, \ldots, 6$, given in Table 1 (see Figures 1 and 2).

Table 1. Darboux polynomials of system (1). $F_{i}=F_{i}(x, y, z)=0$, for $1 \leq i \leq 5$ and $F_{6}=\alpha$, with $\alpha>-a^{2}$ correspond to invariant algebraic surfaces of the Chen system.

\begin{tabular}{ccc}
\hline Case & $a \neq 0, b, c$ & Darboux polynomial \\
\hline (a) & $b=2 a$ & $F_{1}=x^{2}-2 a z$ \\
(b) & $a=-b=c$ & $F_{2}=y^{2}+z^{2}$ \\
(c) & $a=b=-c$ & $F_{3}=2 x^{2}+y^{2}+z^{2}$ \\
(d) & $3 a+c=0$ & $F_{4}=x^{4}+\frac{4}{3} c x^{2} z-\frac{4}{9} c^{2} y^{2}-\frac{8}{9} c^{2} x y-\frac{16}{9} c^{2} x^{2}$ \\
& $b=0$ & \\
(e) & $a+c=0$ & $F_{5}=x^{4}+4 c x^{2} z-4 c^{2} y^{2}+8 c^{2} x y+8 c^{2} x^{2}+48 c^{3} z$ \\
(f) & $b=c=0$ & $F_{6}=y^{2}+z^{2}+2 a z$ \\
\hline
\end{tabular}

Proposition 1.1 will be used in the next sections for studying the global dynamics of system (1). Observe that the invariant algebraic surface

$$
F_{4}=x^{4}+\frac{4}{3} c x^{2} z-\frac{4}{9} c^{2} y^{2}-\frac{8}{9} c^{2} x y-\frac{16}{9} c^{2} x^{2}=0
$$

has a regular part and a singular part (given by the $z$-axis).

In the recent work [Llibre and Valls, 2011] the authors characterized all the the parameter values for which the Chen system (1) has a polynomial first integral. More precisely they proved that system (1) with 


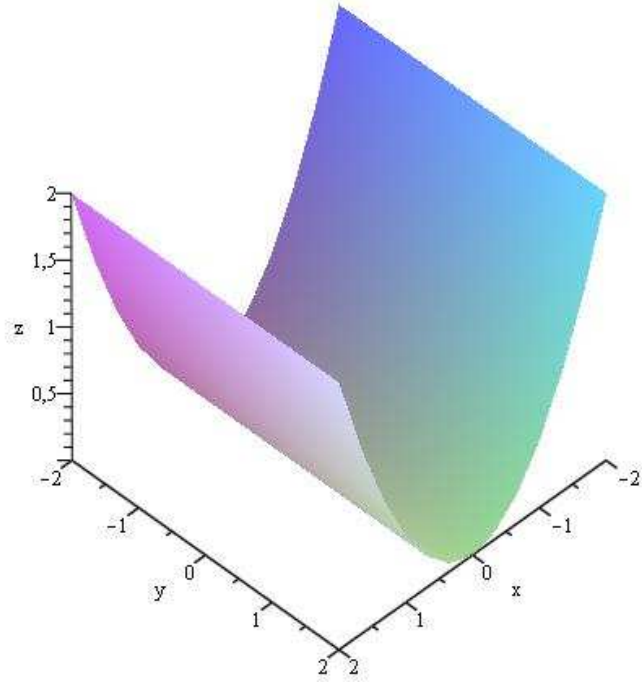

(a)

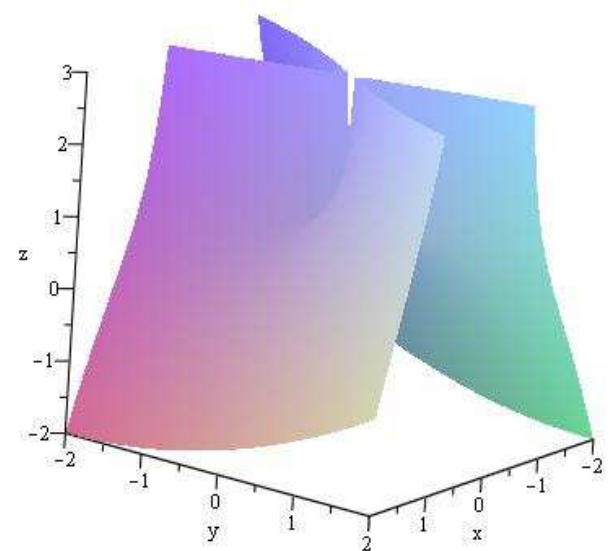

(b)

Fig. 1. Geometrical representation of the invariant algebraic surfaces of the Chen system: (a) $F_{1}=0$, with $a=1$; (b) $F_{4}=0$, with $c=1$.

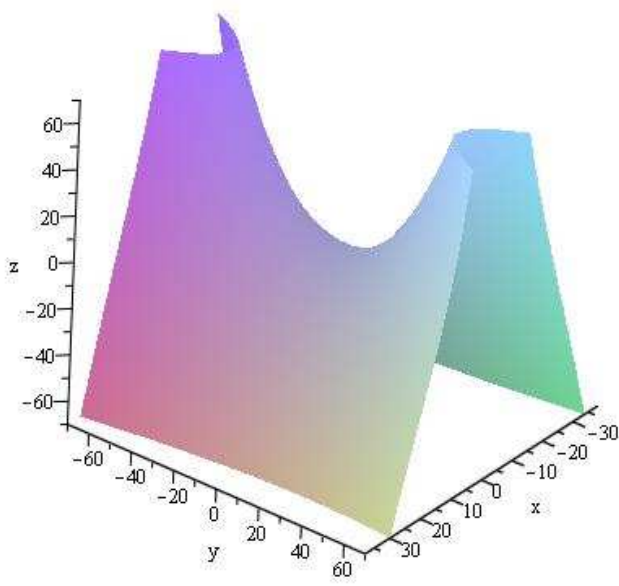

(a)

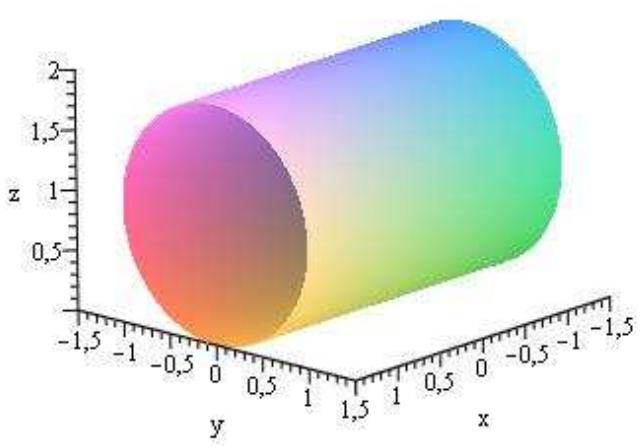

(b)

Fig. 2. Geometrical representation of the invariant algebraic surfaces of the Chen system: (a) $F_{5}=0$, with $c=3$; (b) $F_{6}=0$, with $a=-1$.

$a \neq 0$ has a first integral if and only if $b=c=0$ and, in this case, the first integral is a polynomial in the variable $y^{2}+z^{2}+2 a z$, corresponding to the case (f) of Table 1 . This is an interesting case which shall be 
treated in details in Theorem 1.3 ahead.

As any polynomial differential system, the Chen system (1) can be extended to an analytic system on a closed ball of radius one, whose interior is diffeomorphic to $\mathbb{R}^{3}$ and its boundary, the 2-dimensional sphere $\mathbb{S}^{2}$, plays the role of the infinity. This closed ball is denoted by $\mathbb{D}^{3}$ and called the Poincaré ball, because the technique for doing such an extension is precisely the Poincaré compactification for a polynomial differential system in $\mathbb{R}^{3}$, which is described in details in [Cima and Llibre, 1990] and a summary of it is given in Section 3 ahead. By using this compactification technique the dynamics of system (1) at infinity was studied and we have obtained the following result.

Theorem 1.1. For all values of the parameters $a, b, c$ the phase portrait of system (1) on the sphere at infinity is as shown in Figure 3; i.e. it has two centers at the endpoints of the $x$-axis, the period annulus of these centers end at the circle defined by the infinity of the plane $\{x=0\}$, which is filled of singular points.

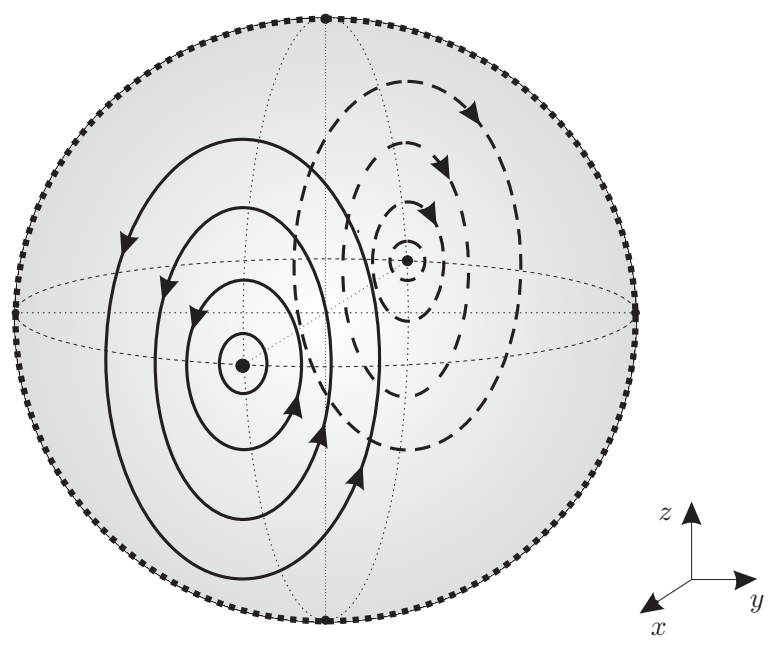

Fig. 3. Phase portrait of system (1) at the infinity of the Poincaré ball (i.e. on the Poincaré sphere $\mathbb{S}^{2}$ ).

It is important to note that the dynamics at infinity does not depend on the parameter values. In this paper we study the dynamics of Chen system (1) in the cases (a), ., (f) given in Table 1 on the whole space $\mathbb{R}^{3}$, including the behavior on the sphere at infinity, that is in the Poincare ball.

The dynamics of Chen system on the invariant algebraic surfaces was studied in [Cao et al., 2008], where all the cases of Proposition 1.1, except the case (f), were considered. In such a work the authors gave a dynamical description of the solutions of system (1) in the projections of the invariant algebraic surfaces $(\mathrm{a}), \ldots$, (e) onto the $(x, y)$-plane. In this way some dynamical aspects are missed. For instance, the dynamics on invariant straight lines and planes, which project orthogonally on the $(x, y)$-plane as points and straight lines respectively, do not appear in the analysis made in [Cao et al., 2008]. Moreover, the authors analyze the dynamics on the invariant surfaces extended to infinity on the Poincaré disc, after the projection of these surfaces on the $x y$-plane. However this analysis does not correspond completely to the analysis of the solutions on the real invariant algebraic surfaces when they are considered in the whole space $\mathbb{R}^{3}$. Also some dynamical objects as heteroclinic orbits, connecting two singular points on a line of singularities, which were numerically detected by us and are described more precisely ahead in this note, were not described in [Cao et al., 2008]. 
The global study of how the invariant algebraic surfaces of the Chen system and the solutions on them reach the infinity, which completes the analysis presented in [Cao et al., 2008], is done here by using the 3dimensional compactification technique of Poincaré. Similar global analysis for other quadratic polynomial differential systems in the compactification of $\mathbb{R}^{3}$ were presented in [Buzzi et al., 2007; Llibre and Messias, 2009; Llibre et al., 2008, 2010; Messias, 2009].

Our main results are stated below.

Theorem 1.2. For all values of the parameters $a, b, c$ the $z$-axis is an invariant set of system (1). The flow in this invariant straight line is as follows: if $b>0$ then the origin $(0,0,0)$ is a global attractor along the $z$-axis; if $b<0$ then the origin is a global repeller; and if $b=0$ all points on the $z$-axis are singular. Furthermore the global phase portraits of the Chen system (1) with $a \neq 0$ and having the invariant algebraic surfaces given in Table 1 are described below.

(a) Assume that $b=2 a$, then system (1) has the invariant algebraic surface $F_{1}=x^{2}-2 a z=0$ (see Figures 1 (a) and 8 for $a<0$ - similar figures take place for $a>0$ ). The boundary at infinity of the surface $F_{1}=0$ is the half great circle $\{x=0, z \leq 0\}$. The finite singular points on $F_{1}=0$ are described in Section 2 , providing 20 different global phase portraits in the Poincaré ball. These phase portraits restricted to the invariant algebraic surface $F_{1}=0$ are described in Figures 9, 10 and 11.

(b) Assume that $a=c=-b$, then system (1) has the invariant algebraic surface $F_{2}=y^{2}+z^{2}=0$, which reduces to the $x$-axis, see Figure 12. The boundary of this surface at infinity is given by the endpoints of the $x$-axis. The origin is the unique finite singular point, providing 2 different global phase portraits on the Poincaré ball. These phase portraits restricted to the invariant algebraic surface $F_{2}=0$ are described in Figure 12.

(c) Assume that $a=b=-c$, then system (1) has the invariant algebraic surface $F_{3}=2 x^{2}+y^{2}+z^{2}=0$, which reduces to the point $(0,0,0)$, see Figure 13. The origin is the only finite singular point, providing 2 different global phase portraits, a global attractor and a global repellor, respectively.

(d) Assume that $3 a+c=0, b=0$, then system (1) has the invariant algebraic surface $F_{4}=x^{4}+4 / 3 c x^{2} z-$ $4 / 9 c^{2} y^{2}-8 / 9 c^{2} x y-16 / 9 c^{2} x^{2}=0$, see Figures 1 (b) and 14. The boundary at infinity of the surface $F_{4}=0$ is the end of the plane $x=0$. The $z$-axis is contained in this surface and it is formed by (nonhyperbolici) singular points of the Chen system. The flow restricted to the surface $F_{4}=0$ is integrable. The phase portrait restricted to the invariant algebraic surface $F_{4}=0$ is described in Figure 14.

(e) Assume that $c=-a$ and $b=4 a$, then system (1) has the invariant algebraic surface $F_{5}=x^{4}+4 c x^{2} z-$ $4 c^{2} y^{2}+8 c^{2} x y+8 c^{2} x^{2}+48 c^{3} z=0$, see Figures $2(a)$ and 15, for $c>0$. The boundary at infinity of the surface $F_{5}=0$ is the end of the plane $x=0$. The origin is the only finite singular point, providing 2 different global phase portraits on the Poincaré ball.

(f) Assume that $b=c=0$, then system (1) has the polynomial first integral $F_{6}=y^{2}+z^{2}+2 a z$ and, consequently, $F_{6}=$ constant is a family of invariant algebraic surfaces, consisting of enclosed cylinders, see Figures 2 (b) and 15-18. The boundary at infinity of the surfaces $F_{6}=$ constant is given by the endpoints of the $x$-axis. The finite singular points are $(0,0, z)$, with $z \in \mathbb{R}$, that is, the $z$-axis is a line of (non-hyperbolic) singularities.

Combining the analytical result stated in case (f) of Theorem 1.2 with an accurate numerical analysis of system (1) with $b=c=0$, which is presented in Section 5 ahead, we can state the following result.

Theorem 1.3. Consider system (1) with $a>0$ and $b=c=0$. In this case the phase space is foliated by 
the invariant cylinders

$$
C_{\alpha}=\left\{(x, y, z) \mid y^{2}+(z+a)^{2}=\alpha+a^{2}, \text { with } x \in \mathbb{R} \text { and } \alpha>-a^{2}\right\}
$$

surrounding the line $\{x \in \mathbb{R}, y=0, z=-a\}$, each one having two singular points, given by the roots of $z^{2}+2 a z-\alpha=0$. One of these singular points is a stable focus (or node) and the other one is a saddle. The 1-dimensional unstable manifolds of the saddle point tend asymptotically to the stable focus (or node), forming two heteroclinic orbits (see Figure $4(a)$ ). Furthermore, the 1-dimensional stable manifolds of the saddle and the orbits contained in the 2-dimensional stable manifold of the focus (or node), except the unstable manifolds of the saddle, are contained in the invariant cylinder $C_{\alpha}$ and go to infinity in backward time, tending asymptotically to the singular points at infinity, located at the endpoints of the $x$-axis (see Figure $4(b))$. Consequently these singular points at infinity are unstable. Furthermore, if $\alpha=-a^{2}$ in (3) we have $y=0, z=-a$, hence system (1) has the invariant straight line $\{x \in \mathbb{R}, y=0, z=-a\}$, containing the singular point $(0,0,-a)$, which is globally stable along this line.

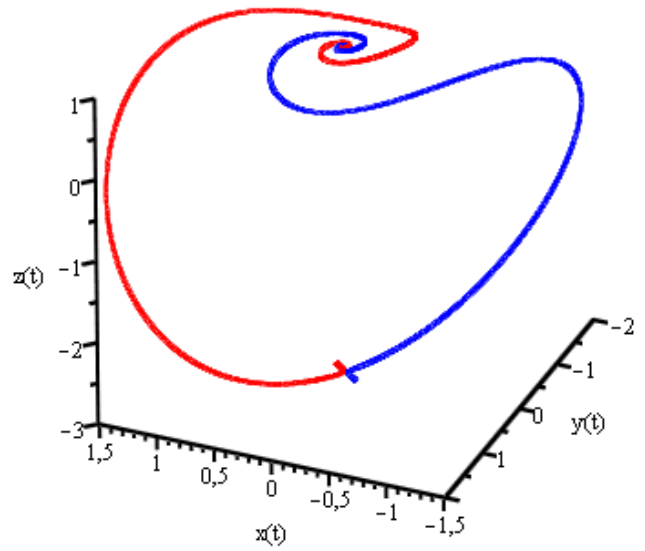

(a)

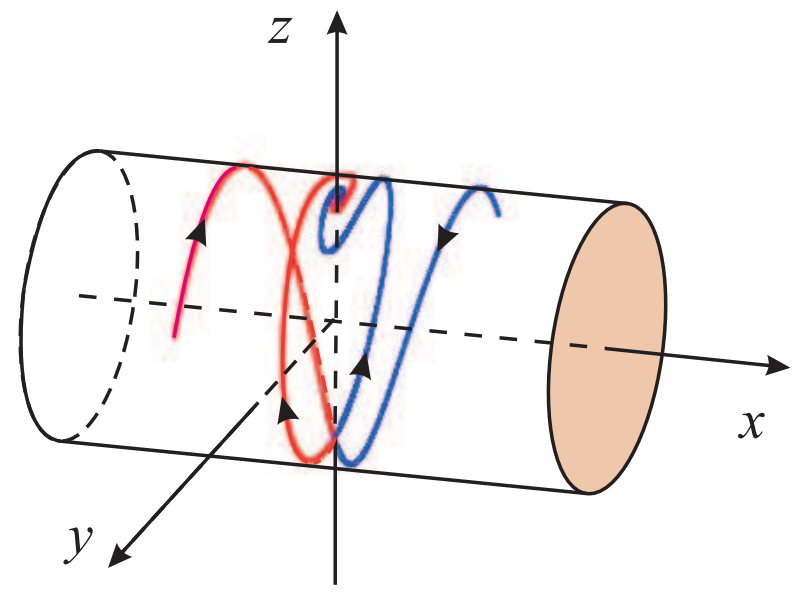

(b)

Fig. 4. (a) Heteroclinic orbits of the Chen system with $b=c=0$ : the unstable manifolds of a saddle connect to a stable focus; (b) Invariant cylinder containing a saddle and a focus with its invariant manifolds. See also Figures 16-18 in Section 5.

From Theorem 1.3, which is proved in Section 4, it follows that system (1) with $a>0$ and $b=c=0$ has a family with infinitely many pairs of heteroclinic orbits, each one contained in one of the cylinders $C_{\alpha}$ given in (3) (see Figures 4 and 15-18). As far as we know this type of heteroclinic orbits, connecting two singular points belonging to a line of singularities, has appeared for the first time in the paper [Kokubu and Roussarie, 2004], where it was called a singularly degenerate heteroclinic cycle. Later on it was shown that this kind of cycles appear in families with infinitely many heteroclinic orbits in the standard Lorenz system [Messias, 2009] and in other quadratic systems [Llibre et al., 2008; Mello et al., 2008; Messias et al., 2008], which also happens for the Chen system as stated in Theorem 1.3. 
We say that a set $V \subseteq \mathbb{D}^{3}$ is invariant by the flow of system (1) if for any $p \in V$ the whole orbit passing through $p$ is contained in $V$. The sphere of the infinity always is an invariant set. Let $\varphi(t)=\varphi(t, p)$ be the solution of the compactified system (1) passing through the point $p \in \mathbb{D}^{3}$, defined on its maximal interval $I_{p}=\mathbb{R}$, because $\mathbb{D}^{3}$ is compact. Then the $\alpha$-limit set of $\varphi$ is the invariant set

$$
\alpha(\varphi)=\left\{q \in \mathbb{D}^{3}: \exists\left\{t_{n}\right\} \text { such that } t_{n} \rightarrow-\infty \text { and } \varphi\left(t_{n}\right) \rightarrow q \text { as } n \rightarrow \infty\right\} .
$$

In a similar way, the $\omega$-limit set of $\varphi$ is the invariant set

$$
\omega(\varphi)=\left\{q \in \mathbb{D}^{3}: \exists\left\{t_{n}\right\} \text { such that } t_{n} \rightarrow \infty \text { and } \varphi\left(t_{n}\right) \rightarrow q \text { as } n \rightarrow \infty\right\} .
$$

A function $I(x, y, z, t)$ is an invariant of system (1) if $d I / d t=0$ on the trajectories of the system. It is easy to see that if $F(x, y, z)$ is a Darboux polynomial of system (1) with constant cofactor $k$, then $I=F e^{-k t}$ is an invariant.

The following theorem gives a complete description of the $\alpha$ - and $\omega$-limit sets in the Poincare ball, including its boundary $\mathbb{S}^{2}$, for the Chen system having the invariant algebraic surfaces of Table 1.

Theorem 1.4. Consider $\varphi(t)=(x(t), y(t), z(t)), t \in \mathbb{R}$, an orbit through the point $p \in \mathbb{D}^{3}$ of the Chen system (1) satisfying one of the conditions (a),..,(e) of Table (1). Then the $\alpha$-and $\omega$-limit sets of $\varphi(t)$, denoted by $\alpha(\varphi)$ and $\omega(\varphi)$, respectively, are contained in the set $\left\{F_{i}=0\right\} \cup \mathbb{S}^{2}$. More specifically, if the cofactor $k_{i}>0$ then we have the following possibilities:

(a) $p \in \mathbb{S}^{2} \Rightarrow \alpha(\varphi), \omega(\varphi) \subset \mathbb{S}^{2}$

(b) $p \in\left(\mathbb{D}^{3} \backslash \mathbb{S}^{2}\right) \Rightarrow \alpha(\varphi) \subset \overline{\left\{F_{i}=0\right\}}$;

(c) $p \in\left(\mathbb{D}^{3} \backslash \mathbb{S}^{2}\right), F_{i}(p)=0 \Rightarrow \omega(\varphi) \subset \overline{\left\{F_{i}=0\right\}}$;

(d) $p \in\left(\mathbb{D}^{3} \backslash \mathbb{S}^{2}\right), F_{i}(p) \neq 0 \Rightarrow \omega(\varphi) \subset \mathbb{S}^{2}$;

and if $k_{i}<0$ then

(e) $p \in \mathbb{S}^{2} \Rightarrow \alpha(\varphi), \omega(\varphi) \subset \mathbb{S}^{2}$;

(f) $p \in\left(\mathbb{D}^{3} \backslash \mathbb{S}^{2}\right) \Rightarrow \omega(\varphi) \subset \overline{\left\{F_{i}=0\right\}}$;

(g) $p \in\left(\mathbb{D}^{3} \backslash \mathbb{S}^{2}\right), F_{i}(p)=0 \Rightarrow \alpha(\varphi) \subset \overline{\left\{F_{i}=0\right\}}$;

(h) $p \in\left(\mathbb{D}^{3} \backslash \mathbb{S}^{2}\right), F_{i}(p) \neq 0 \Rightarrow \alpha(\varphi) \subset \mathbb{S}^{2}$.

If system (1) satisfies the condition (f) of Table 1 and $\varphi(t)=(x(t), y(t), z(t))$ is an orbit through the point $p \in \mathbb{D}^{3}$ with $F_{6}(p)=\alpha$, then the whole orbit $\varphi(t), t \in \mathbb{R}$ is contained in $F_{6}=\alpha$.

The aim of this paper is to prove the results stated above. It is organized as follows. In Section 2 we summarize the results related to the dynamics and bifurcation of finite singularities. In Section 3 we prove Theorem 1.1 by using the Poincaré compactification for a polynomial vector field in $\mathbb{R}^{3}$. In Section 4 we prove Theorems 1.2, 1.4 and 1.3. Based on the analytical results proved, we performed a numerical study of system (1), which is presented in Section 5.

\section{Dynamics of finite singularities}

We start this section with the proof of Proposition 1.1, which is due to Lu and Zhang [Lu and Zhang, 2007]. 


\subsection{Proof of Proposition 1.1}

If $a=0$, Chen system (1) becomes

$$
\dot{x}=0, \quad \dot{y}=c x-x z+c y, \quad \dot{z}=x y-b z .
$$

Thus $H(x, y, z)=x$ is a first integral and the flow on each level $H^{-1}(h)$ is determined by the linear differential system

$$
\dot{y}=c h-h z+c y, \quad \dot{z}=h y-b z .
$$

For $a \neq 0$ we have $\nabla F_{i} \cdot(P, Q, R)=k_{i} F_{i}$, where each $F_{i}, i=1, \ldots, 6$, is a Darboux polynomial with corresponding cofactor $k_{i}$, and they are given by:

(a) $F_{1}(x, y, z)=x^{2}-2 a z$ and $k_{1}(x, y, z)=-2 a$;

(b) $F_{2}(x, y, z)=y^{2}+z^{2}$ and $k_{2}(x, y, z)=2 a$;

(c) $F_{3}(x, y, z)=2 x^{2}+y^{2}+z^{2}$ and $k_{3}(x, y, z)=-2 a$;

(d) $F_{4}(x, y, z)=x^{4}+\frac{4}{3} c x^{2} z-\frac{4}{9} c^{2} y^{2}-\frac{8}{9} c^{2} x y-\frac{16}{9} c^{2} x^{2}$ and $k_{4}(x, y, z)=4 / 3 c$;

(e) $F_{5}(x, y, z)=x^{4}+4 c x^{2} z-4 c^{2} y^{2}+8 c^{2} x y+8 c^{2} x^{2}+48 c^{3} z$ and $k_{5}(x, y, z)=4 c$.

Observe that all the cofactors are constants.

In what follows we shall study the local stability at the singular points of the Chen system for the parameters satisfying the conditions of Table 1. It is obvious that in each case the singular points are contained in the invariant algebraic surfaces $F_{i}=0, i=1, \ldots, 5$ and $F_{6}=\alpha$, with $\alpha>-a^{2}$.

\subsection{Singular points in the case $b=2 a$}

Consider Chen system (1) satisfying the case (a) of Table 1 , that is $b=2 a$ and $a \neq 0$. Then we have the following possibilities.

\subsubsection{Subcase $2 c-a=0$}

The unique singular point is $(0,0,0)$. The eigenvalues are

$$
\lambda_{1}=-2 a, \quad \lambda_{2}=\frac{c-a+\sqrt{\Delta}}{2}, \quad \lambda_{3}=\frac{c-a-\sqrt{\Delta}}{2},
$$

where $\Delta=c^{2}+6 a c-3 a^{2}$. We have

(a1) If $a>0$ then $\lambda_{1}<0, \lambda_{2}=0$ and $\lambda_{3}<0$;

(a1') If $a<0$ then then $\lambda_{1}>0, \lambda_{2}>0$ and $\lambda_{3}=0$.

\subsubsection{Subcase $(2 c-a) a<0$}

The only singular point is $(0,0,0)$. The eigenvalues satisfies the following:

(b1) If $a<0, \Delta \geq 0$ and $c-a>0$, then $\lambda_{1}>0, \lambda_{2}>0$ and $\lambda_{3}>0$;

(b2) If $a<0, \Delta \geq 0$ and $c-a<0$, then $\lambda_{1}>0, \lambda_{2}<0$ and $\lambda_{3}<0$;

(b3) If $a<0, \Delta<0$ and $c-a>0$, then $\lambda_{1}>0, \lambda_{2}=u+i v$ and $\lambda_{3}=u-i v$ with $u, v>0$;

(b4) If $a<0, \Delta<0$ and $c-a<0$, then $\lambda_{1}>0, \lambda_{2}=-u+i v$ and $\lambda_{3}=-u-i v$ with $u, v>0$;

(b1') If $a>0, \Delta \geq 0$ and $c-a>0$, then $\lambda_{1}<0, \lambda_{2}>0$ and $\lambda_{3}>0$;

(b2') If $a>0, \Delta \geq 0$ and $c-a<0$, then $\lambda_{1}<0, \lambda_{2}<0$ and $\lambda_{3}<0$; 
(b3') If $a>0, \Delta<0$ and $c-a>0$, then $\lambda_{1}<0, \lambda_{2}=u+i v$ and $\lambda_{3}=u-i v$ with $u, v>0$;

(b4') If $a>0, \Delta<0$ and $c-a<0$, then $\lambda_{1}<0, \lambda_{2}=-u+i v$ and $\lambda_{3}=-u-i v$ with $u, v>0$.

The cases $\Delta \leq 0, c-a=0$ and $\Delta>0, c-a=0$ are impossible if $(2 c-a) a<0$.

\subsubsection{Subcase $(2 c-a) a>0$}

There are 3 singular points

$$
(0,0,0), \quad \text { and } \quad( \pm \sqrt{2 a(2 c-a)}, \pm \sqrt{2 a(2 c-a)}, 2 c-a) .
$$

The eigenvalues associated to $(0,0,0)$ are classified according the following conditions:

(c1) If $a<0, \Delta \geq 0$ and $c-a>0$, then $\lambda_{1}>0, \lambda_{2}>0$ and $\lambda_{3}<0$;

(c2) If $a<0, \Delta>0$ and $c-a=0$, then $\lambda_{1}>0, \lambda_{2}>0$ and $\lambda_{3}<0$;

(c3) If $a<0, \Delta \geq 0$ and $c-a<0$, then $\lambda_{1}>0, \lambda_{2}>0$ and $\lambda_{3}<0$;

(c4) If $a<0, \Delta<0$ and $c-a>0$, then $\lambda_{1}>0, \lambda_{2}=u+i v$ and $\lambda_{3}=u-i v$ with $u, v>0$;

(c5) If $a<0, \Delta<0$ and $c-a<0$, then $\lambda_{1}>0, \lambda_{2}=-u+i v$ and $\lambda_{3}=-u-i v$ with $u, v>0$;

(c1') If $a>0, \Delta \geq 0$ and $c-a>0$, then $\lambda_{1}<0, \lambda_{2}>0$ and $\lambda_{3}<0$;

(c2') If $a>0, \Delta>0$ and $c-a=0$, then $\lambda_{1}<0, \lambda_{2}>0$ and $\lambda_{3}<0$;

(c3') If $a>0, \Delta \geq 0$ and $c-a<0$, then $\lambda_{1}<0, \lambda_{2}>0$ and $\lambda_{3}<0$;

(c4') If $a>0, \Delta<0$ and $c-a>0$, then $\lambda_{1}<0, \lambda_{2}=u+i v$ and $\lambda_{3}=u-i v$ with $u, v>0$;

(c5') If $a>0, \Delta<0$ and $c-a<0$, then $\lambda_{1}<0, \lambda_{2}=-u+i v$ and $\lambda_{3}=-u-i v$ with $u, v>0$.

The eigenvalues associated to $( \pm \sqrt{2 a(2 c-a)}, \pm \sqrt{2 a(2 c-a)}, 2 c-a)$ are

$$
\eta_{1}=-2 a, \quad \eta_{2}=\frac{c-a+\sqrt{\Delta_{1}}}{2}, \quad \eta_{3}=\frac{c-a-\sqrt{\Delta_{1}}}{2},
$$

where $\Delta_{1}=c^{2}-18 a c+9 a^{2}$. The eigenvalues satisfy the following:

(d1) If $a<0, \Delta_{1} \geq 0, c-a>0$, then $\eta_{1}>0, \eta_{2}>0$ and $\eta_{3}>0$;

(d2) If $a<0, \Delta_{1} \geq 0, c-a<0$, then $\eta_{1}>0, \eta_{2}<0$ and $\eta_{3}<0$;

(d3) If $a<0, \Delta_{1}<0, c-a<0$, then $\eta_{1}>0, \eta_{2}=-u+i v$ and $\eta_{3}=-u-i v$ with $u, v>0$;

(d4) If $a<0, \Delta_{1}<0, c-a>0$, then $\eta_{1}>0, \eta_{2}=u+i v$ and $\eta_{3}=u-i v$ with $u, v>0$;

(d5) If $a<0, \Delta_{1}<0, c-a=0$, then $\eta_{1}>0, \eta_{2}=i v$ and $\eta_{3}=-i v$ with $v>0$;

(d1') If $a>0, \Delta_{1} \geq 0, c-a>0$, then $\eta_{1}<0, \eta_{2}>0$ and $\eta_{3}>0$;

(d2') If $a>0, \Delta_{1} \geq 0, c-a<0$, then $\eta_{1}<0, \eta_{2}<0$ and $\eta_{3}<0$;

(d3') If $a>0, \Delta_{1}<0, c-a<0$, then $\eta_{1}<0, \eta_{2}=-u+i v$ and $\eta_{3}=-u-i v$ with $u, v>0$;

(d4') If $a>0, \Delta_{1}<0, c-a>0$, then $\eta_{1}<0, \eta_{2}=u+i v$ and $\eta_{3}=u-i v$ with $u, v>0$;

(d5') If $a>0, \Delta_{1}<0, c-a=0$, then $\eta_{1}>0, \eta_{2}=i v$ and $\eta_{3}=-i v$ with $v>0$.

The case $\Delta_{1} \geq 0, c-a=0$ is impossible for $a \neq 0$.

\subsection{Singular points in the case $a=-b=c$}

The only singular point is $(0,0,0)$. The corresponding eigenvalues are $\lambda_{1}=-a, \lambda_{2}=a$ and $\lambda_{3}=a$. Thus we have only two cases:

(a1) If $a>0$ then $\lambda_{1}<0$ and $\lambda_{2}=\lambda_{3}>0$;

(a2) If $a<0$ then $\lambda_{1}>0$ and $\lambda_{2}=\lambda_{3}<0$. 


\subsection{Singular points in the case $a=b=-c$}

The only singular point is $(0,0,0)$. The corresponding eigenvalues are $\lambda_{1}=-a, \lambda_{2}=-a+\sqrt{2 a^{2}} i$ and $\lambda_{3}=-a-\sqrt{2 a^{2}} i$. Thus we have only two cases:

(a1) If $a>0$ then $\lambda_{1}<0, \lambda_{2}=-u+i v$ and $\lambda_{3}=-u-i v$ with $u, v>0$;

(a2) If $a<0$ then $\lambda_{1}>0, \lambda_{2}=u+i v$ and $\lambda_{3}=u-i v$ with $u, v>0$.

\subsection{Singular points in the case $b=0, c=-3 a$}

The singular point are $\left(0,0, z_{0}\right), z_{0} \in \mathbb{R}$. The corresponding eigenvalues are $\lambda_{1}=0, \lambda_{2}=-2 a+\sqrt{-3 a^{2}-a z}$ and $\lambda_{3}=-2 a-\sqrt{-3 a^{2}-a z}$. Thus we have the following cases:

(a1) If $a>0, z_{0}<-7 a$ then $\lambda_{2}>0$ and $\lambda_{3}<0$;

(a2) If $a>0, z_{0}=-7 a$ then $\lambda_{2}=0$ and $\lambda_{3}<0$;

(a3) If $a>0,-7 a<z_{0}<-3 a$ then $\lambda_{2}<0$ and $\lambda_{3}<0$;

(a4) If $a>0, z_{0}=-3 a$ then $\lambda_{2}<0$ and $\lambda_{3}<0$;

(a5) If $a>0, z_{0}>-3 a$ then $\lambda_{2}=-u+i v$ and $\lambda_{3}=-u-i v$ with $u, v>0$;

(a1') If $a<0, z_{0}>-7 a$ then $\lambda_{2}>0$ and $\lambda_{3}<0$;

(a2') If $a<0, z_{0}=-7 a$ then $\lambda_{2}>0$ and $\lambda_{3}=0$;

(a3') If $a<0,-3 a<z_{0}<-7 a$ then $\lambda_{2}>0$ and $\lambda_{3}>0$;

(a4') If $a<0, z_{0}=-3 a$ then $\lambda_{2}>0$ and $\lambda_{3}>0$;

(a5') If $a<0, z_{0}<-3 a$ then $\lambda_{2}=u+i v$ and $\lambda_{3}=u-i v$ with $u, v>0$.

\subsection{Singular points in the case $b=4 a, c=-a$}

The only singular point is $(0,0,0)$. The corresponding eigenvalues are $\lambda_{1}=-4 a, \lambda_{2}=-a+\sqrt{2 a^{2}} i$ and $\lambda_{3}=-a-\sqrt{2 a^{2}} i$. Thus we have only two cases:

(a1) If $a>0$ then $\lambda_{1}<0, \lambda_{2}=-u+i v$ and $\lambda_{3}=-u-i v$ with $u, v>0$;

(a2) If $a<0$ then $\lambda_{1}>0, \lambda_{2}=u+i v$ and $\lambda_{3}=u-i v$ with $u, v>0$.

\subsection{Singular points in the case $b=c=0$}

System (1) has a line of singularities contained in the $z$-axis. The corresponding eigenvalues are

$$
\lambda_{1}=\frac{-a+\sqrt{a^{2}-4 a(a+z)}}{2}, \quad \lambda_{2}=\frac{-a-\sqrt{a^{2}-4 a(a+z)}}{2} \text { and } \quad \lambda_{3}=0 .
$$

Consider $a>0$. Then the singular point $(0,0, z)$ is locally a saddle point if $z<-a$, a stable focus if $z>-(3 / 4) a$ and a stable node if $-a<z \leq-(3 / 4) a$. The singular point $(0,0,-a)$ is more degenerate: it has two zero eigenvalues; there exists an invariant line parallel to the $x$-axis passing through $(0,0,-a)$ and this point is a global attractor along this line, in the case $a>0$. In fact, for $z=-a$ the Chen system (1) reduces to

$$
\dot{x}=-a, \quad \dot{y}=0 \quad \dot{z}=0 .
$$

This case will be better analyzed in Section 4 . 


\section{Poincaré compactification and the proof of Theorem 1.1}

In this section we present the formulas concerning the Poincaré compactification for a polynomial differential system in $\mathbb{R}^{3}$ with degree 2 . Poincaré introduced this compactification for polynomial vector fields in $\mathbb{R}^{2}$. Its extension to $\mathbb{R}^{n}$ for $n>2$ can be found in [Cima and Llibre, 1990] and some applications in [Llibre et al., 2008, 2010]. More precisely we consider the polynomial differential system

$$
\dot{x}=P(x, y, z), \quad \dot{y}=Q(x, y, z), \quad \dot{z}=R(x, y, z),
$$

or equivalently its associated polynomial vector field $X=(P, Q, R)$. This polynomial system is extended to an analytic system on a closed ball of radius one, whose interior is diffeomorphic to $\mathbb{R}^{3}$ and its boundary, the 2-dimensional sphere $\mathbb{S}^{2}$; plays the role of the infinity. This closed ball is denoted by $\mathbb{D}^{3}$ and called the Poincaré ball. We consider 6 open charts on $\mathbb{S}^{2}$

(a) $U_{1}=\{(x, y, z): x>0\}$ and $V_{1}=\{(x, y, z): x<0\}$,

(b) $U_{2}=\{(x, y, z): y>0\}$ and $\left.V_{2}=\{(x, y, z)): y<0\right\}$,

(c) $U_{3}=\{(x, y, z): z>0\}$ and $V_{3}=\{(x, y, z): z<0\}$.

The phase portrait on $U_{1}$ is the central projection of the phase portrait of the system

$$
\dot{u}=w^{2}(-u P+Q), \quad \dot{v}=w^{2}(-v P+R),
$$

where $u, v$ are the coordinates of the tangent plane $T \mathbb{S}_{(1,0,0)}^{2}$ at $(1,0,0) \in \mathbb{S}^{2}$, and $P, Q$ and $R$ are the polynomial functions evaluated at $(1 / w, u / w, v / w)$. Moreover we make $w=0$ in order to get the points at the infinite sphere (see figure 5 ).

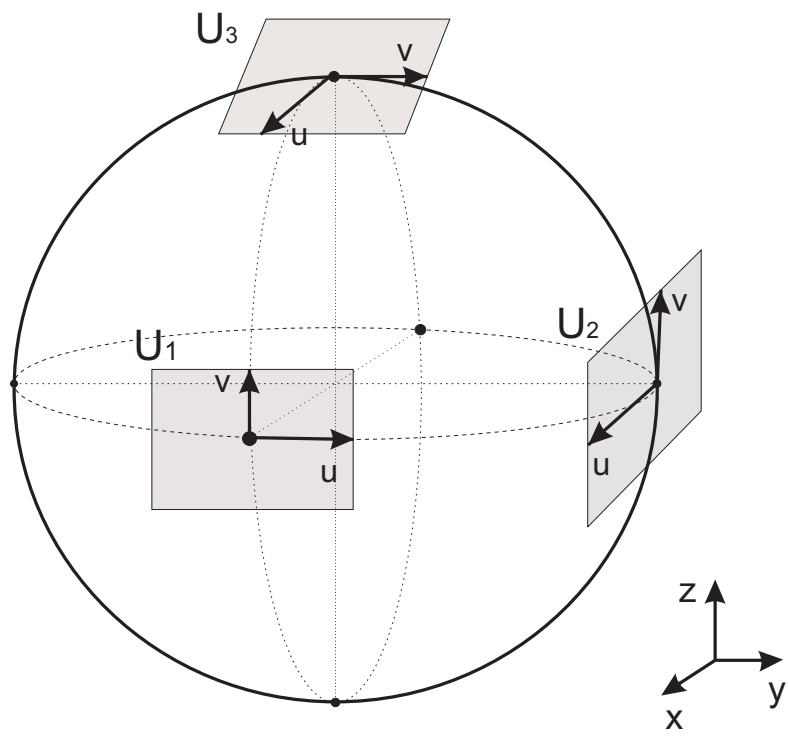

Fig. 5. Local charts related to the Poincar compactification: $U_{1}: y=\frac{u}{w}, z=\frac{v}{w} ; U_{2}: x=\frac{u}{w}, z=\frac{v}{w} ;$ and $U_{3}: x=\frac{u}{w}, y=\frac{v}{w}$. The charts $V_{1}, V_{2}$ and $V_{3}$ are diametrically opposed.

The flow on $U_{2}$ is determined by the system

$$
\dot{u}=w^{2}(-u Q+P), \quad \dot{v}=w^{2}(-v Q+R),
$$


where $u, v$ are the coordinates of the tangent plane $T \mathbb{S}_{(0,1,0)}^{2}$ at $(0,1,0) \in \mathbb{S}^{2}$, and the polynomial functions $P, Q$ and $R$ are evaluated at $(u / w, 1 / w, v / w)$. Moreover we made $w=0$ in order to get the points at the infinite sphere.

The flow on $U_{3}$ is determined by the system

$$
\dot{u}=w^{2}(-u R+P), \quad \dot{v}=w^{2}(-v R+Q),
$$

where $u, v$ are the coordinates of the tangent plane $T \mathbb{S}_{(0,0,1)}^{2}$ at $(0,0,1) \in \mathbb{S}^{2}$, and $P, Q$ and $R$ are evaluated at $(u / w, v / w, 1 / w)$. Moreover we consider $w=0$.

The expression for the extend differential system in the local chart $V_{i}, i=1,2,3$ is the same as in $U_{i}$ multiplied by $(-1)$.

\subsection{Proof of Theorem 1.1}

From system (4) the expression of the Poincaré compactification of system (1) in the local chart $U_{1}$ is given by

$$
\begin{aligned}
& \dot{u}=-a u^{2} w+a u w+c w-a w-v+c u w \\
& \dot{v}=-a u v w+a v w+u-b v w .
\end{aligned}
$$

For $w=0$ (which corresponds to the points on the sphere $\mathbb{S}^{2}$ of the infinity) (7) reduces to

$$
\dot{u}=-v, \quad \dot{v}=u,
$$

from which follows that system (1) has the canonical linear center in the local chart of the Poincaré sphere which has the endpoint of the $x$-axis at the origin. The flow in the local chart $V_{1}$ is the same as the flow in the local chart $U_{1}$ reversing appropriately the time, since the compactified vector field in $V_{1}$ coincides with the vector field in $U_{1}$ multiplied by -1 . Hence system (1) also has the canonical linear center in the local chart $V_{1}$ of the Poincaré sphere. See figure 1.

From system (5) the expression of the Poincaré compactification of system (1) in the local chart $U_{2}$ is given by

$$
\begin{aligned}
& \dot{u}=-c u^{2} w+a u^{2} w+u^{2} v-c u w+a w-a u w, \\
& \dot{v}=-c u v w+a u v w+u v^{2}-c v w+u-b v w .
\end{aligned}
$$

For $w=0$ (which corresponds to the points on the sphere $\mathbb{S}^{2}$ of the infinity) (9) reduces to

$$
\dot{u}=u^{2} v, \quad \dot{v}=u v^{2}+u .
$$

System (10) is integrable, since $H(u, v)=\frac{v^{2}+1}{u^{2}}$ is a first integral. The phase portrait has the $v$-axis formed by singular points. As before, the flow in the local chart $V_{2}$ is the same as the flow in the local chart $U_{2}$ reversing appropriately the time. See figure 7.

From system (6) the expression of the Poincaré compactification of system (1) in the local chart $U_{3}$ is given by

$$
\begin{aligned}
& \dot{u}=-u^{2} v+b u w+a v w-a u w \\
& \dot{v}=-u v^{2}+b v w+c u w-a u w-u+c v w .
\end{aligned}
$$

For $w=0$ (which corresponds to the points on the sphere $\mathbb{S}^{2}$ of the infinity) (11) reduces to

$$
\dot{u}=-u^{2} v, \quad \dot{v}=-u v^{2}-u .
$$


System (12) is equal to system (10), reversing the time hence its phase space is as shown in figure 7. As before, the flow in the local chart $V_{3}$ is the same as the flow in the local chart $U_{3}$ reversing appropriately the time.

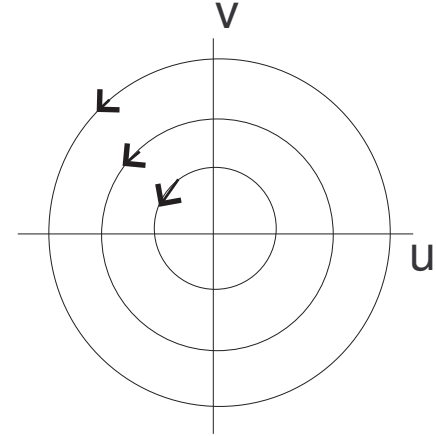

U1

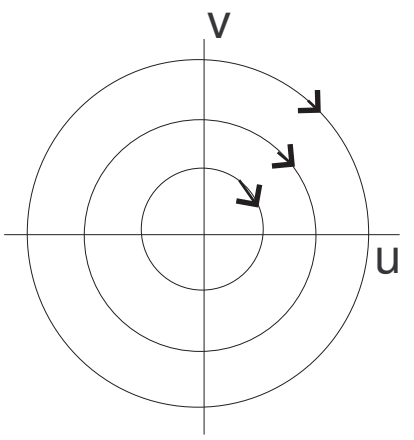

V1

Fig. 6. Dynamics of system (1) on the sphere of the infinity in the local charts $U_{1}$ and $V_{1}$.

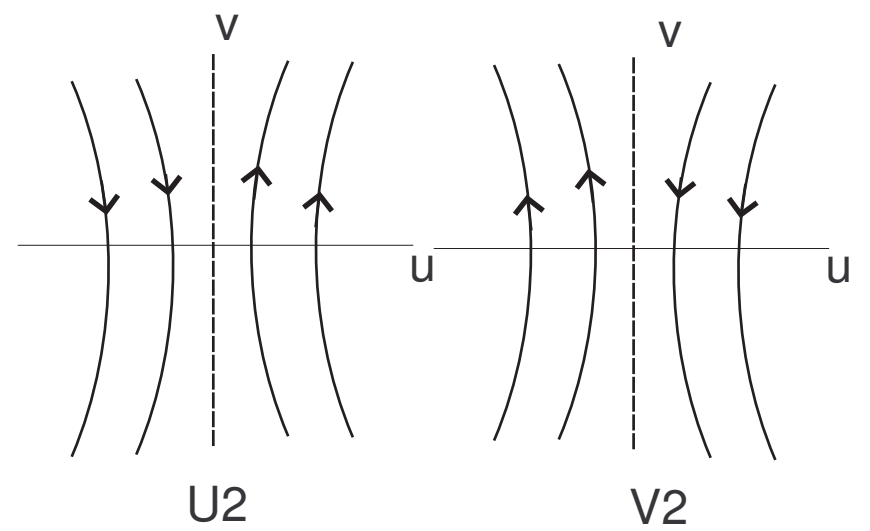

Fig. 7. Dynamics of system (1) on the sphere of the infinity in the local charts $U_{2}$ and $V_{2}$. The $v$-axis is filled of singular points.

\section{The proofs of Theorems $1.2,1.3$ and 1.4}

The following result, which will be used through this section, is proved in [Llibre et al., 2008].

Lemma 4.1. Let $f(x, y, z)=0$ be an algebraic surface of $\mathbb{R}^{3}$ of degree $m$. The extension of this surface to the boundary of the Poincaré ball is contained in the curve defined by

$$
w^{m} f\left(\frac{x}{w}, \frac{y}{w}, \frac{z}{w}\right)=0, \quad w=0
$$




\subsection{Proof of Theorem 1.2}

Denote $\mathcal{Z}=\{(0,0, z): z \in \mathbb{R}\}$ the $z$-axis. For any $\left(0,0, z_{0}\right) \in \mathcal{Z}$ the solution of the Chen system (1) is $\varphi(t)=\left(0,0, z_{0} e^{-b t}\right)$. Thus the whole orbit passing through $\left(0,0, z_{0}\right)$ is contained in $\mathcal{Z}$. Moreover if $b>0$ then $\lim _{t \rightarrow+\infty} \varphi(t)=(0,0,0)$, and if $b<0$ then $\lim _{t \rightarrow-\infty} \varphi(t)=(0,0,0)$. This proves the first part of the statement of Theorem 1.2. The cases (a), ., (f) are treated below.

Case (a) In this case the invariant algebraic surface is $F_{1}=x^{2}-2 a z=0$ where $a \neq 0$ and $b=2 a$. Then the graphic of the invariant algebraic surface $x^{2}-2 a z=0$ is a parabolic cylinder filled by the parallel parabolas $z=x^{2} / 2 a$. The singular points of Chen system are contained in this surface. Suppose that $a<0$. This parabolic cylinder in the Poincaré ball $\mathbb{D}^{3}$ is a surface for which the endpoint of each parabola is the point $(x, y, z)=(0,0,-1)$ of the sphere $\mathbb{S}^{2}$ at infinity.

According to Lemma 4.1 the boundary of the surface on the sphere $\mathbb{S}^{2}$ of the infinity is given by the system

$$
x^{2}-2 a y w=0, \quad w=0 .
$$

Simplifying the expression we get $x=0$. It means that the boundary at infinity of the surface $F_{1}=0$ is the half great circle $x=0, z \leq 0$. For $a>0$ we have an analogous result.

A complete analysis of the dynamics of the Chen system on the parabolic cylinders $z=x^{2} / 2 a$ projected onto the $(x, y)$-plane can be found in [Cao et al., 2008]. In Table 2 we have the classification of the singular points.

Table 2. Singular points of system (1) on the surface $x^{2}-2 a z=0$.

\begin{tabular}{cccccc}
\hline$c-a$ & $\Delta_{1}$ & $\Delta$ & $a(2 c-a)$ & Singularities & Figure \\
\hline$=0$ & & & \multicolumn{4}{c}{ s saddle, 2 centers } & $(9)$ \\
$\neq 0$ & & & 1 unstable node & $(9)$ \\
$<0$ & & $\geq 0$ & $<0$ & 1 stable node \\
$>0$ & & $\geq 0$ & $<0$ & 1 unstable node \\
$<0$ & & $<0$ & $<0$ & 1 stable focus \\
$>0$ & & $<0$ & $<0$ & 1 unstable focus \\
$<0$ & $\geq 0$ & & $>0$ & 1 saddle, 2 stable nodes \\
$>0$ & $\geq 0$ & & $>0$ & 1 saddle, 2 unstable nodes \\
$<0$ & $<0$ & & $>0$ & 1 saddle, 2 stable foci \\
$>0$ & $<0$ & & $>0$ & 1 saddle, 2 unstable foci
\end{tabular}

Case (b) In this case the invariant algebraic surface is $F_{2}=y^{2}+z^{2}=0$, whose graphic is the $x$-axis. There is only one singular point of Chen system and it is contained in this surface. Suppose that $a>0$. The boundary of the surface on the sphere $\mathbb{S}^{2}$ of the infinity is formed by the two centers $( \pm 1,0,0)$. The singular point is stable.

Case (c) In this case the invariant algebraic surface is $F_{3}=x^{2}+y^{2}+z^{2}=0$, which reduces to the origin $(0,0,0)$.

Theorem 1.4 implies that if $a>0$ then the related cofactor is negative and $(0,0,0)$ is a global attractor; and if $a<0$ then related cofactor is positive and then $(0,0,0)$ is a global repellor. 


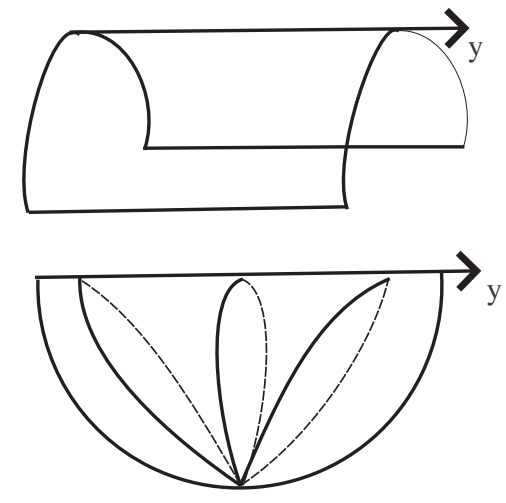

(a)

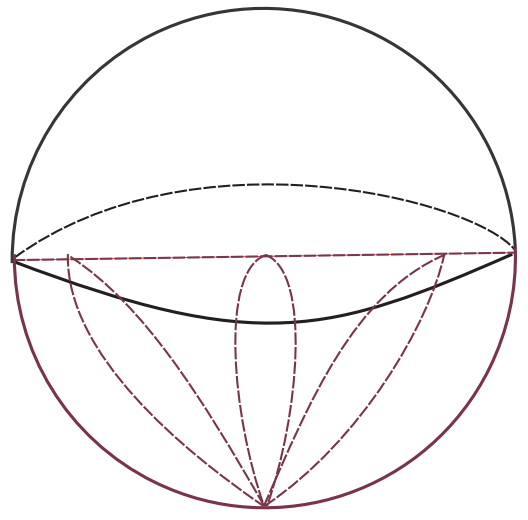

(b)

Fig. 8. The invariant algebraic surface $x^{2}-2 a z=0$ with $a<0$ in $\mathbb{R}^{3}$ (a) and its compactification to the Poincaré ball (b).
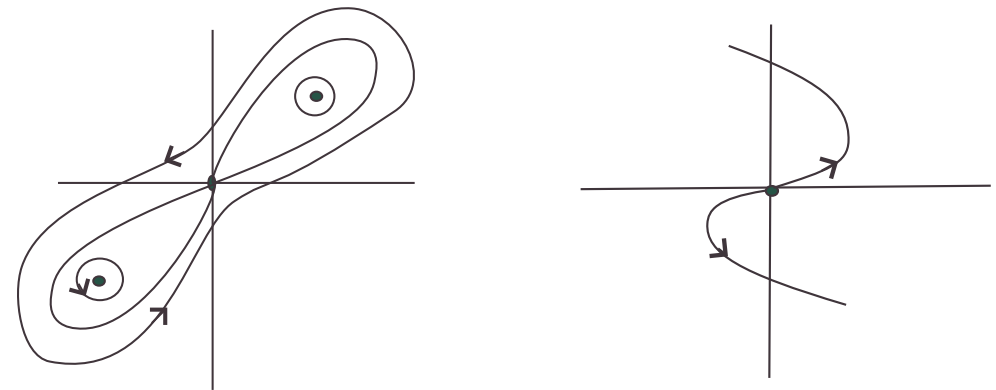

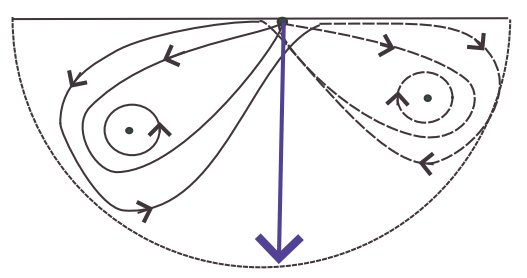

(a)

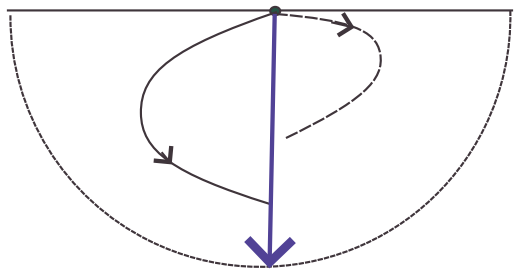

(b)

Fig. 9. Dynamics of the Chen system on the invariant algebraic surface $x^{2}-2 a z=0$ with $a<0$ and $c-a=0$ (a) and $c-a>0, \Delta \geq 0, a(2 c-a)<0(\mathrm{~b})$.

Case (d) In this case the invariant algebraic surface is

$$
F_{4}=x^{4}+\frac{4}{3} c x^{2} z-\frac{4}{9} c^{2} y^{2}-\frac{8}{9} c^{2} x y-\frac{16}{9} c x^{2}=0 .
$$

The boundary of this surface on the sphere $\mathbb{S}^{2}$ of the infinity is the great circle $x=0$ and the two endpoints of the $z$-axis. A complete analysis of the dynamics of the Chen system on the invariant algebraic surface projected onto the $(x, y)$-plane can be found in [Cao et al., 2008]. They proved that the projected system has the first integral $H(x, y)=x^{2}+4 a^{2} y^{2} x^{2}-8 a^{2} y x$, which completely determine the phase portrait. Furthermore, there exists a line of singularities contained in the $z$-axis. The singular point $(0,0, z)$ is a saddle normally hyperbolic to the $z$-axis if $z<-7 a$; it is a normally hyperbolic stable node if 

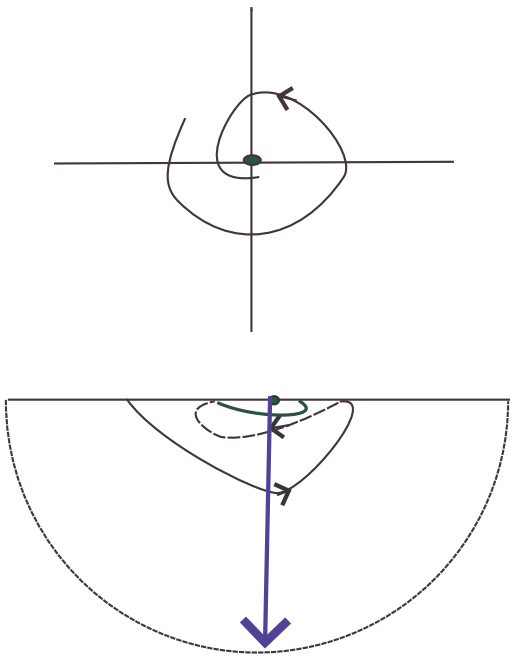

(a)
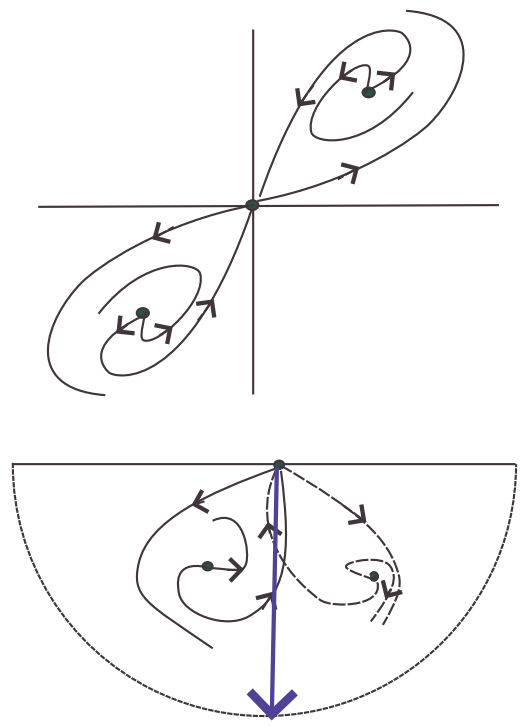

(b)

Fig. 10. Dynamics of the Chen system on the invariant algebraic surface $x^{2}-2 a z=0$ with $a<0$ and (a) $c-a>0, \Delta<0$, $a(2 c-a)<0$ and (b) $c-a<0, \Delta_{1} \geq 0, a(2 c-a)>0$.
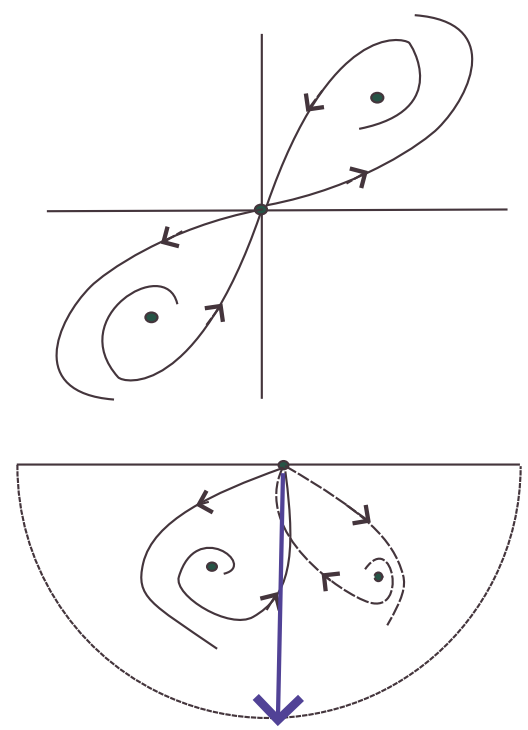

Fig. 11. Dynamics of the Chen system on the invariant algebraic surface $x^{2}-2 a z=0$ with $a<0$ and $c-a>0, \Delta_{1}<0$, $a(2 c-a)>0$.

$-7 a \leq z \leq-3 a$; and it is a normally hyperbolic stable focus if $z>-3 a$.

Case (e) In this case the invariant algebraic surface is

$$
F_{5}=x^{4}+4 c x^{2} z-4 c^{2} y^{2}+8 c^{2} x y+8 c^{2} x^{2}+48 c^{3} z=0 .
$$

The boundary of this surface on the sphere $\mathbb{S}^{2}$ of the infinity is the great circle $\{x=0\}$. The singular point $(0,0,0)$ belongs to this surface. A complete analysis of the dynamics of the Chen system on the invariant 


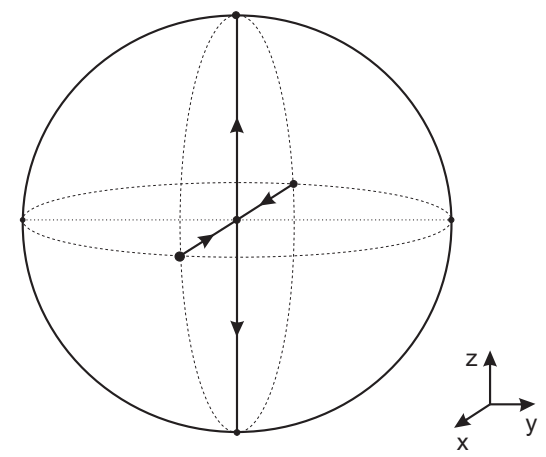

Fig. 12. Dynamics of the Chen system on the invariant algebraic surface $y^{2}+z^{2}=0$ with $a<0$. The bold line is the $z$-axis, which is also invariant.

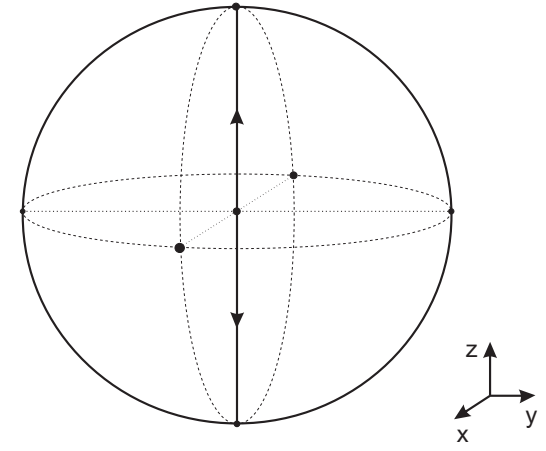

Fig. 13. Dynamics of the Chen system on the invariant algebraic surface $x^{2}+y^{2}+z^{2}=0$. The bold line is the $z$-axis, which is also invariant.

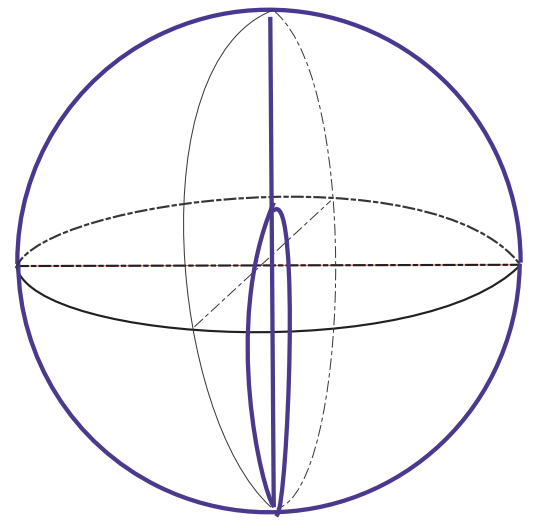

(a)

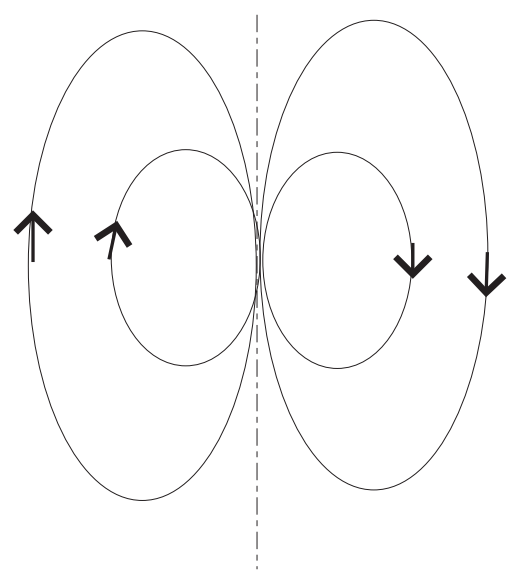

(b)

Fig. 14. Invariant algebraic surface $x^{4}+\frac{4}{3} c x^{2} z-\frac{4}{9} c^{2} y^{2}-\frac{8}{9} c^{2} x y-\frac{16}{9} c^{2} x^{2}=0$ on the Poincaré ball, for $c>0$ and the projected phase portrait.

algebraic surface projected onto the $(x, y)$-plane can be found in [Cao et al., 2008]. They proved that if $c<0$ then the projected system has a stable focus at the origin, while for $c>0$ the projected system has 
an unstable focus at the origin.

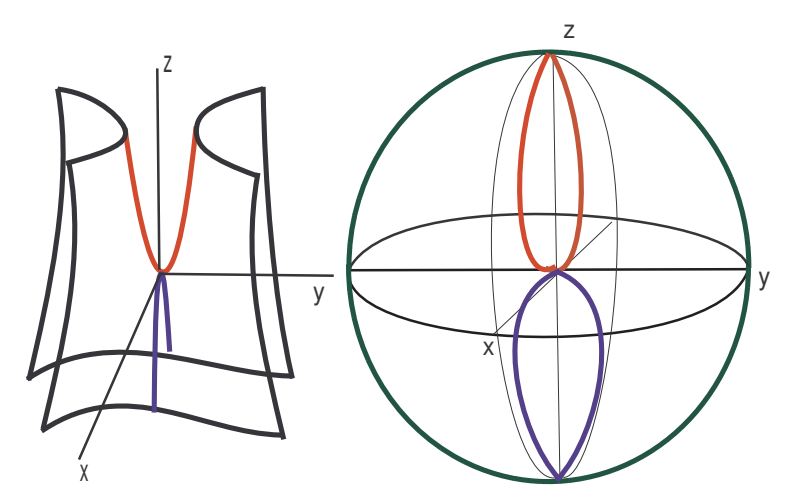

Fig. 15. Invariant algebraic surface $x^{4}+4 c x^{2} z-4 c^{2} y^{2}+8 c^{2} x y+8 c^{2} x^{2}+48 c^{3} z=0$, for $c>0$, in $\mathbb{R}^{3}$ and in the Poincaré ball.

Case (f) In this case the invariant algebraic surfaces are given by $F_{6}=y^{2}+z^{2}+2 a z=\alpha$, with $\alpha>-a^{2}$, which consists of a family of invariant cylinders surrounding the line $\{(x, y, z) \mid y=0$ and $z=-a\}$. The boundary of these cylinders on the sphere $\mathbb{S}^{2}$ of the infinity consist of the endpoints of the $x$-axis. There is a line of singularities at the $z$-axis (the normal hyperbolicity of these singular points are studied in Subsections 2.6 and 4.2). This completes the proof of Theorem 1.2.

\subsection{Proof of Theorem 1.3}

For $b=c=0$ and $a>0$ system (1) reduces to

$$
\dot{x}=a(y-x), \quad \dot{y}=-a x-x z, \quad \dot{z}=x y,
$$

which has the first integral $F(x, y, z)=y^{2}+z^{2}+2 a z$, since

$$
(\nabla F) \cdot(P, Q, R)=(0,2 y, 2 z+2 a) \cdot(a y-a x,-a x-x z, x y)=0 .
$$

Consequently the cylinders

$$
C_{\alpha}=\left\{(x, y, z) \mid y^{2}+(z+a)^{2}=\alpha+a^{2}, \text { with } x \in \mathbb{R} \text { and } \alpha>-a^{2}\right\},
$$

surrounding the invariant straight line $\{x \in \mathbb{R}, y=0, z=-a\}$ are invariant algebraic surfaces of system (13). This system has the singular points $(0,0, z), z \in \mathbb{R}$, i.e. the $z$-axis is filled of singular points. Then in each invariant cylinder $C_{\alpha}$ there exist two singular points, given by the intersection of $C_{\alpha}$ with the $z$-axis, which are given by the roots of

$$
z^{2}+2 a z-\alpha=0, \quad \alpha>-a^{2} .
$$

We study the local (normal) stability of these singular points. The Jacobian matrix of system (13) at the singular point $(0,0, z)$ is given by

$$
\left(\begin{array}{ccc}
-a & a & 0 \\
-z-a & 0 & 0 \\
0 & 0 & 0
\end{array}\right)
$$


which has the eigenvalues

$$
\lambda_{1,2}=-\frac{a}{2} \pm \frac{\sqrt{a^{2}-4 a(a+z)}}{2}, \quad \lambda_{3}=0 .
$$

Then the singular point $(0,0, z)$ is locally a normally hyperbolic saddle if $z<-a$ and it is locally a normally hyperbolic stable focus or node if $z>-a$ (a node if $-a<z \leq-(3 / 4) a$ and a focus if $z>-(3 / 4) a$ ). Note that for $z=-a$ and $y=0$ system (13) reduces to

$$
\dot{x}=-a x, \quad \dot{y}=0, \quad \dot{z}=0,
$$

which has the invariant straight line $\{x \in \mathbb{R}, y=0, z=-a\}$ containing the singular point $(0,0,-a)$, which is globally stable along this line.

The invariant straight line $\{x \in \mathbb{R}, y=0, z=-a\}$ and the invariant cylinders $C_{\alpha}$ have their boundaries at infinity as the negative and positive endpoints of the $x$-axis, which by the considerations above are unstable singular points. The invariant cylinders $C_{\alpha}$ does not contain periodic orbits of system (13). In fact, isolating $y$ from the expression of $C_{\alpha}$ and substituting it in system (13) we have that the flow restricted to $C_{\alpha}$ is given by

$$
\begin{aligned}
& \dot{x}=-a x \pm a \sqrt{\alpha+a^{2}-(z+a)^{2}}, \\
& \dot{z}= \pm x \sqrt{\alpha+a^{2}-(z+a)^{2}},
\end{aligned}
$$

from which we obtain

$$
\dot{x}=-a x+\frac{a \dot{z}}{x}
$$

or, equivalently,

$$
\frac{d}{d t}\left(\frac{1}{2} x^{2}-a z\right)=-a x^{2} .
$$

Suppose that $(x(t), z(t))$ is a periodic solution of system (15). Then the function

$$
\frac{1}{2} x(t)^{2}-a z(t)
$$

is also periodic, hence its derivative must assume positive and negative values. But from (16) it follows that this derivative takes only negative values, which is a contradiction. Therefore system (13) has no periodic orbits on the cylinders $C_{\alpha}$.

From the considerations above, applying the Poincaré-Bendixson Theorem for the flow defined on the cylinders $C_{\alpha}$, it follows that the 1-dimensional unstable manifolds of a saddle point must tend asymptotically to a stable focus (or node), having this point as their $\omega$-limit set, forming two heteroclinic orbits (see Figure 4). Furthermore, the 1-dimensional stable manifolds of the saddle and the orbits contained in the 2-dimensional stable manifold of the focus (or node), except the unstable manifolds of the saddle, are contained in the invariant cylinder and go to infinity in backward time, tending asymptotically to the singular points at infinity, located at the endpoints of the $x$-axis, thus having these points as their $\alpha$-limit sets (see again Figure 4).

The proof of Theorem 1.3 is complete.

\subsection{Proof of Theorem 1.4}

Suppose that $k_{i}<0$. Consider $q \in \omega(\varphi)$. Suppose that $q$ is not in $\mathbb{S}^{2}$. Thus there exists $t_{n} \rightarrow \infty$ and $c \in \mathbb{R}$ such that $\varphi\left(t_{n}\right) \rightarrow q, F_{i}\left(\varphi\left(t_{n}\right)\right)=\frac{c}{e^{-k_{i} t_{n}}} \rightarrow 0$ and $F_{i}\left(\varphi\left(t_{n}\right)\right) \rightarrow F_{i}(q)$. It follows that $F_{i}(q)=0$ and then 
$q \in\left\{F_{i}=0\right\}$. Suppose now that $q \in \alpha(\varphi)$ and $q$ is not in $\mathbb{S}^{2}$. Thus there exists $t_{n} \rightarrow-\infty$ and $c \in \mathbb{R}$ such that $\varphi\left(t_{n}\right) \rightarrow q$ and $F_{i}\left(\varphi\left(t_{n}\right)\right) \cdot e^{-k_{i} t_{n}} \rightarrow F_{i}(q) \cdot 0=c$. Thus $c=0$ and then $F_{i}(\varphi(t)) \cdot e^{-k_{i} t}=0$, for any $t \in \mathbb{R}$. It implies that $F_{i}(\varphi(t))=0$, for any $t \in \mathbb{R}$. Analogously we prove the case $k_{i}>0$.

\section{Numerical simulations}

From the cases described in Theorem 1.2 we can highlight the case (f), for which system (1) has a line of singularities contained in the $z$-axis. As stated in Theorem 1.3, the phase space is foliated by the invariant cylinders $y^{2}+z^{2}+2 a z=\alpha$, with $\alpha>-a^{2}$, each one having two singular points of system (1). One of these singular points is a stable focus (or node) and the other one is a saddle. The numerical simulations performed indicate clearly that the unstable 1-dimensional manifolds of a saddle connect to a stable focus, forming a heteroclinic trajectory (see Figure 4); on the other hand, the 1-dimensional stable manifolds of the saddle and the orbits contained in the 2-dimensional stable manifold of the focus (or node), except the unstable manifolds of the saddle, go to infinity in backward time (see again Figure 4). In the Figures 16 to 18 we present some more numerical solutions of system (1) with $b=c=0$ and $a>0$, confirming the statements of Theorem 1.3.

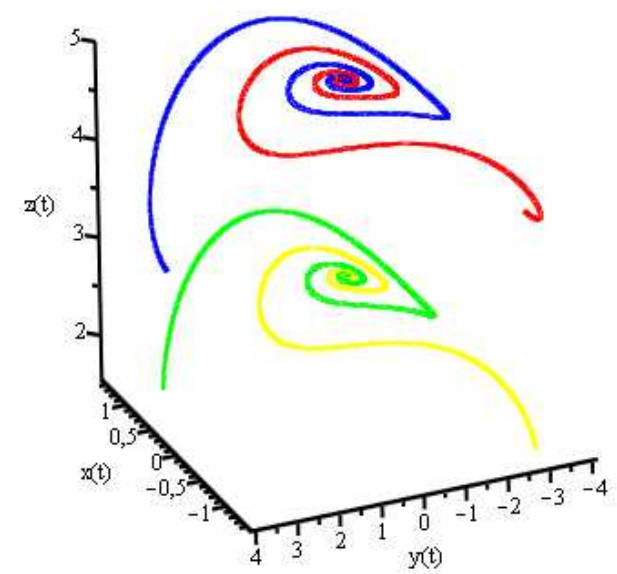

(a)

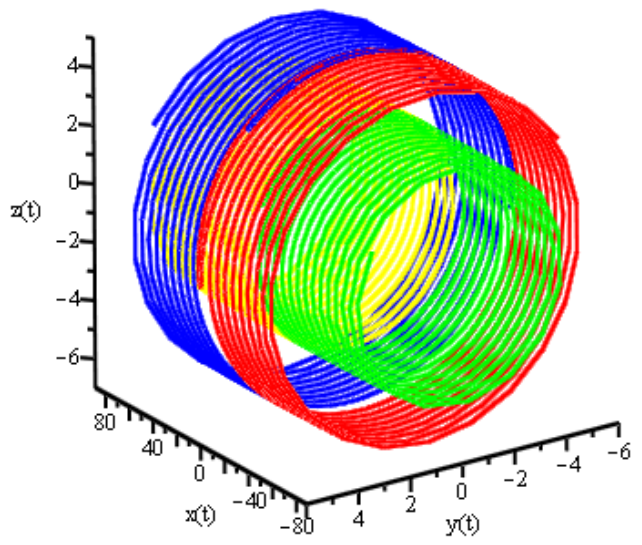

(b)

Fig. 16. (a) Two stable foci of system (1) with $b=c=0$ and $a>0$ contained on two different invariant cylinders shown in (b), obtained following the solutions in (a) backwards in time.

The family of cylinders described above shrink into the invariant straight line $\{y=0, z=-a\}$, where the dynamics is trivial as it was described in Section 2.

\section{Acknowledgements}

The first author is partially supported by the MICINN/FEDER grant MTM2008-03437, the Generalitat de Catalunya grant 2009SGR-410 and ICREA Academia. The second author is supported by CNPq-Brazil 


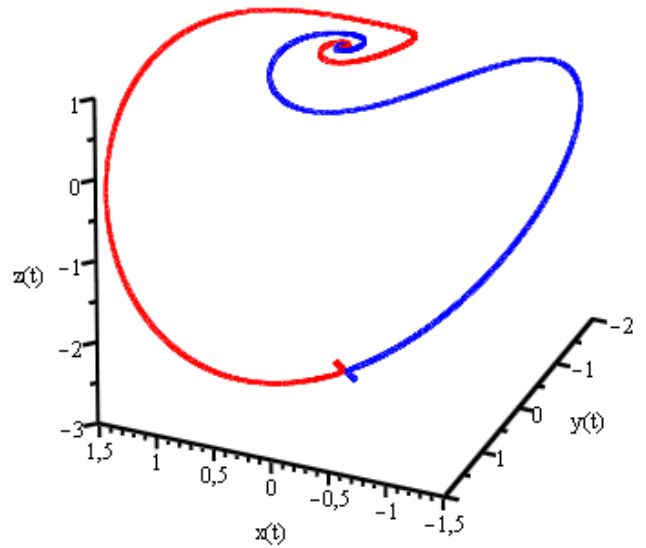

(a)

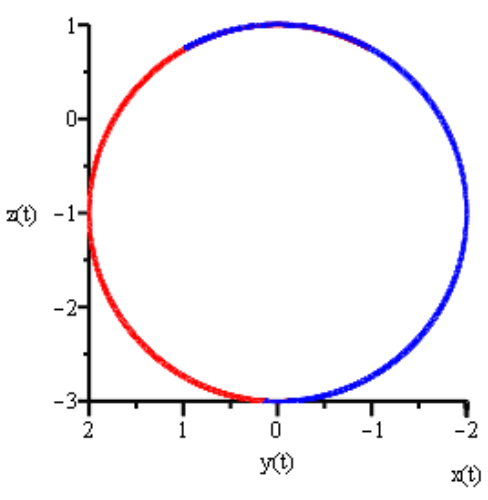

(b)

Fig. 17. (a) Two heteroclinic orbits connecting a saddle point with a stable focus; (b) its projection onto the $y z-$ plane, showing that they are contained on the invariant cylinder given by $F_{6}=\alpha$.

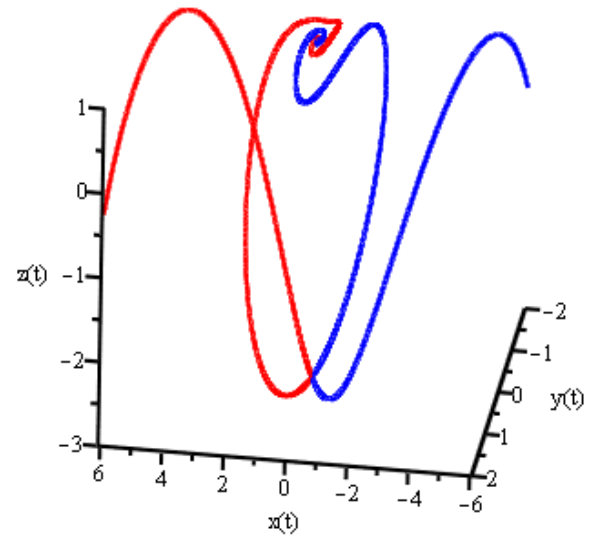

(a)

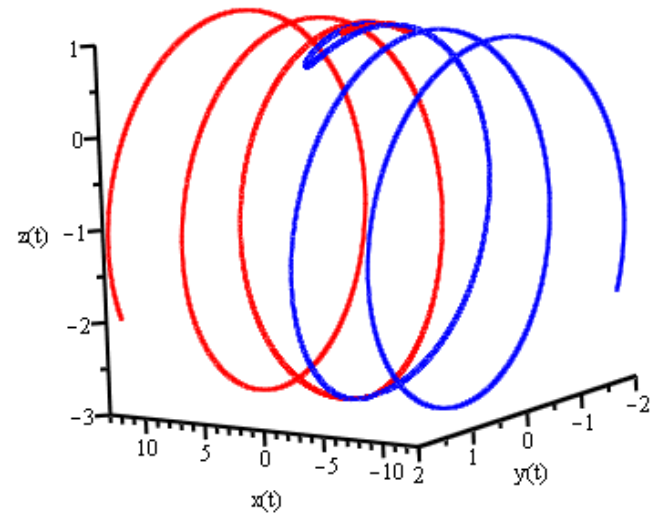

(b)

Fig. 18. (a) Following in backward time the stable manifolds of a saddle shown in Figure 17 (a), showing that these manifolds are contained on a cylinder $F_{6}=\alpha$.

under the project 305204/2009-2. The third author is partially supported by CNPq and FAPESP. All the authors are supported by the Int. Coop. Proj. CAPES/MECD-TQED II and PHB-2009-0025. 


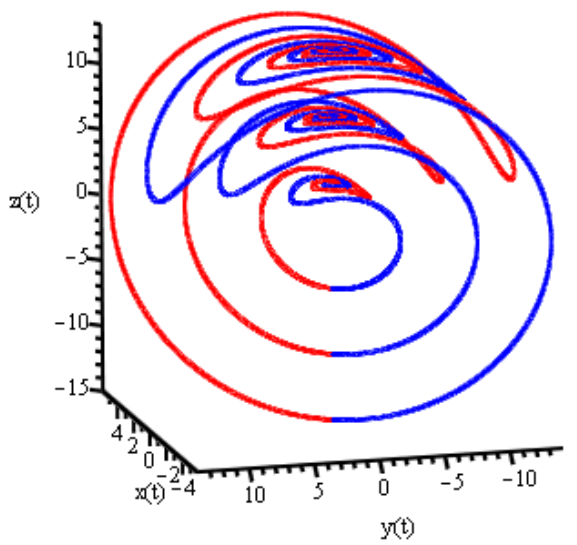

(a)

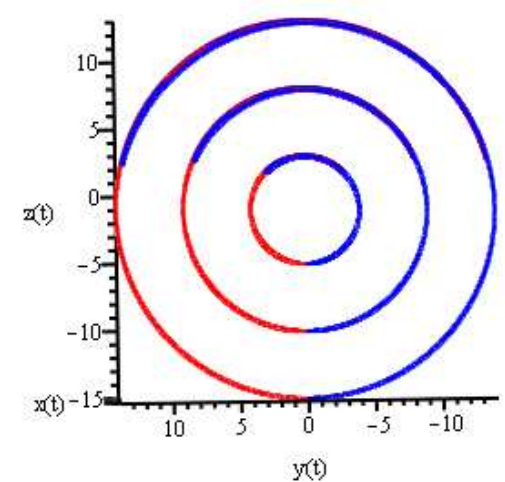

(b)

Fig. 19. Three pairs of heteroclinic orbits connecting saddle points on the $z$-axis to stable foci; (b) its projection onto the $y z$-plane, showing that they are contained on three different invariant cylinders given by $F_{6}=\alpha$ with different values of $\alpha$.

\section{References}

Buzzi, C. A., Llibre, J. and Medrado, J. C. [2007] "Periodic orbits for a class of reversible quadratic vector field on $\mathbb{R}^{3}, "$ J. Math. Anal. Appl. 335, 1335-1346.

Cao, J., Chen, C. and Zhang, X. [2008] "The Chen system having an algebraic surface," Internat. J. Bifur. Chaos Appl. Sci. Engrg. 18, 3753-3758.

Chen, G. and Ueta, T. [1999] "Yet another chaotic attractor," Internat. J. Bifur. Chaos Appl. Sci. Engrg. 9, 14651466.

Cima, A. and Llibre, J. [1990] "Bounded polynomial vector fields," Trans. Amer. Math. Soc. 318, 557-579.

Kokubu, H. and Roussarie, R. [2004] "Existence of a singularly degenerate heteroclinic cycle in the Lorenz system and its dynamical consequences: Part i," J. Dyn. Diff. Eq. 16, 513-557.

Llibre, J. and Messias, M. [2009] "Global dynamics of the Rikitake system," Physica D: Nonlinear Phenomena 238(3), 241-252.

Llibre, J., Messias, M. and da Silva, P. R. [2008] "On the global dynamics of the Rabinovich system," J. Phys. A: Math. Theor. 41(275210), pp 21.

Llibre, J., Messias, M. and da Silva, P. R. [2010] "Global dynamics of the Lorenz system with invariant algebraic surfaces," Internat. J. Bifur. Chaos Appl. Sci. Engrg. 20, 3137-3155.

Llibre, J. and Valls, C. [2011] "Polynomial first integrals for the Chen and lorenz systems," to appear in Internat. J. Bifur. Chaos Appl. Sci. Engrg .

Lorenz, E. N. [1963] "Deterministic nonperiodic flow," J. Atmos. Sci. 20, 1302-141.

Lu, J., Chen, G. and Celikovsky, S. [2002] "Bridge the gap between the Lorenz system and the Chen system," Internat. J. Bifur. Chaos Appl. Sci. Engrg. 12, 2917-2926.

Lu, T. and Zhang, X. [2007] "Darboux polynomials and algebraic integrability of the Chen system," Internat. J. Bifur. Chaos Appl. Sci. Engrg. 17, 2739-2748.

Mello, L. F., Messias, M. and Braga, D. C. [2008] "Bifurcation analysis of a new Lorenz-like chaotic system," Chaos, 
Solitons and Fractals 37, 1244-1255.

Messias, M. [2009] "Dynamics at infinity and the existence of singularly degenerate heteroclinic cycles in the Lorenz system," J. Phys. A: Math. Theor. 42(115101), pp 18.

Messias, M., Nespoli, C. and Dalbelo, T. M. [2008] "Mechanics for the creation of strange attractors in Rossler's second system," (Portuguese) TEMA Tend. Mat. Apl. Comput. 9, 275-285. 